Instituto Internacional de Investigación y Desarrollo Tecnológico Educativo INDTEC, C.A.

DOI: https://doi.org/10.29394/Scientific.issn.2542-2987.2020.5.16.7.141-161

OAI-PMH: http://www.indteca.com/ojs/index.php/Revista Scientific/oai

Artículo Original / Original Article

\title{
Relación entre personalidad y desempeño académico de adolescentes en instituciones educativas públicas y privadas
}

Autor: Luís Leonardo Zambrano Vacacela Universidad Nacional de Educación, UNAE

leozamv@hotmail.com

Azogues, Ecuador

https://orcid.org/0000-0002-2968-1351

\section{Resumen}

El objetivo del estudio es determinar la relación entre factores de personalidad y el desempeño académico de adolescentes en instituciones educativas públicas y privadas. La investigación se fundamentó en un enfoque cuantitativo no experimental con diseño transeccional causal, para esto se aplicó el Cuestionario de Personalidad para Adolescentes (HSPQ) a 136 discentes de 13 a 16 años pertenecientes a noveno grado de Educación General Básica en establecimientos fiscales y particulares del sector rural en la ciudad de Cuenca-Ecuador. Los resultados revelan que las mujeres tienden hacia la sumisión, responsabilidad y trabajo grupal, mientras que los hombres a la irresponsabilidad, agresividad e incumplimiento de normas, adicionalmente, existe correlación mínima entre la personalidad y el desempeño académico y, finalmente el alumnado de instituciones privadas alcanza mejores calificaciones en el promedio general que el estudiantado de establecimientos fiscales.

Palabras clave: personalidad; adolescente; educación básica.

Cómo citar este artículo:

Zambrano, L. (2020). Relación entre personalidad y desempeño académico de adolescentes en instituciones educativas públicas y privadas. Revista Scientific, 5(16), 141-161, e-ISSN: 2542-2987. Recuperado de: https://doi.org/10.29394/Scientific.issn.2542-2987.2020.5.16.7.141-161

Fecha de Recepción: 20-12-2019
Fecha de Aceptación:

23-03-2020
Fecha de Publicación: 05-05-2020 


\title{
Relationship between personality and academic performance of adolescents in public and private educational institutions
}

\begin{abstract}
The objective of the study is to determine the relationship between personality factors and the academic performance of adolescents in public and private educational institutions. The research was based on a nonexperimental quantitative approach with a causal transectional design, for this the Personality Questionnaire for Adolescents (HSPQ) was applied to 136 students from 13 to 16 years old belonging to the ninth grade of Basic General Education in tax and private establishments of the rural sector in the city of Cuenca-Ecuador. The results reveal that women tend towards submission, responsibility and group work, while men tend to irresponsibility, aggressiveness and non-compliance with norms. Additionally, there is a minimal correlation between personality and academic performance and, finally, students from private institutions. achieves better grades in the general average than the students of tax establishments.
\end{abstract}

Keywords: personality; youth; basic education.

How to cite this article:

Zambrano, L. (2020). Relationship between personality and academic performance of adolescents in public and private educational institutions. Revista Scientific, 5(16), 141-161, e-ISSN: 2542-2987. Recovered from: https://doi.org/10.29394/Scientific.issn.2542-2987.2020.5.16.7.141-161

Date Received: 20-12-2019
Date Acceptance:

23-03-2020
Date Publication: 05-05-2020 


\section{Introducción}

La adolescencia desde su conceptualización resulta difícil concretar, en este sentido, Güemes-Hidalgo, González-Fierro y Hidalgo (2017a): la definen como la transición entre infancia adultez acompañada de transmutaciones físicas, psicológicas, emocionales y sociales. Por su lado, Jensen y Nutt (2019): afirman que este concepto va más allá de la semántica, filosofía o psicología, porque ser adolescente repercute al propio individuo, padres, educadores y hasta al sistema judicial.

La Organización Mundial de la Salud (OMS), la ubica en edades de 10 a 19 años y según la Sociedad Americana de Salud y Medicina de la Adolescencia (SAHM), desde los 10 a los 21 años, con relación a esto, Güemes-Hidalgo, González-Fierro y Hidalgo (2017b): afirman que, a pesar de haber discrepancias sobre el comienzo y terminación del período, todos coinciden que es una etapa medular y trascendental en la existencia humana.

Actualmente, de acuerdo con investigaciones de criminología desarrolladas por Alarcón, Pérez-Luco, Wenger, Salvo y Chesta (2018): los profesionales de la educación aducen que en las instituciones los adolescentes presentan conductas agresivas y antisociales reiteradas, además existe evidencia que señala que un pequeño grupo continúa con ese tipo de comportamiento en la adultez.

Es por lo que autores como Morán, Urchaga, Manga y Fínez (2018a): mencionan que la etapa de la adolescencia es trascendental para construir la personalidad. Además, revelan que este constructo es moldeado por las relaciones interpersonales entre iguales y paternales. De la misma manera, Malo-Cerrato, Martín-Perpiñá y Viñas-Poch (2018): explican que las interacciones y relaciones entre la familia, amigos o desconocidos en la web, influyen sobre las concepciones, comportamientos y la personalidad.

Desde esta perspectiva se deduce que la personalidad es la forma de ser de cada adolescente, determina su comportamiento, actitudes y 
emociones, caracteriza o diferencia a un individuo de otro, se coincide con Guerra (2015a): quien explica que la personalidad es el conjunto de particularidades que definen a los seres humanos, depende de las emociones e incurre sobre la conducta.

\subsection{Clasificación de la personalidad}

Sobre la organización de este constructo, Eysenck (1992): parte de bases fisiológicas y biológicas y lo divide en tres factores: neurocentrísmocontrol, introversión-extroversión, como los más importantes y psicotismo como menos trascendente.

Por otro lado, dentro de la personalidad existen factores que marcan diferencias entre las personas. Tal es el caso que para Catell y Catell (1995a), existe un: "reservado-abierto; calmoso-excitable; sumiso-dominante; sobrioentusiasta; despreocupado-consciente; cohibido-emprendedor; segurodubitativo; sereno-aprensivo; sociable-autosuficiente; más-menos integrado y relajado-tenso" (pág. 24).

Por su lado, Cloninger (2002): de acuerdo con similitudes y diferencias, se divide la personalidad en tipos y rasgos. Entre estas manifestaciones se localiza el gobernante, de quien consigue, el evasivo y el socialmente útil. Sin embargo, hay quienes fragmentan la personalidad en factores a partir de hábitos, emociones, actitudes y pensamientos relacionados con el entorno. En este sentido, Benítez, Sánchez y Serrano (2015a): plantea identidad, humor, estado de ánimo, conductas contradictorias e influencia del grupo.

\subsection{Factores generales de la personalidad}

Entre los factores generales existentes en la estructura de la personalidad, Catell y Catell (1995b), mencionan: "ajuste-ansiedad, introversión-extraversión, calma-excitabilidad y dependencia-independencia" (pág. 24); que se encuentran comprendidos en el High School Personality 
Questtionnarie (HSPQ) o Cuestionario de Personalidad para Adolescentes adaptado para su aplicación en el año 95.

\subsubsection{Ajuste-ansiedad}

El término ansiedad proviene del latín anxietas, que significa zozobra, inquietud, en medicina quiere decir angustia que acompaña a las enfermedades, en otras palabras, para Sierra, Ortega y Zubeidat (2003): es la mezcla de distintas emociones manifestadas de manera física o psicológica ante la presencia inminente del peligro.

En el ámbito educativo, según Contreras, Espinosa, Esguerra, Haikal, Polanía y Rodríguez (2005): la ansiedad está relacionada directamente con el desempeño académico, influye concisamente en los estudiantes, aunque esto depende de la orientación hacia el aprendizaje desarrollado por agentes externos. Bajo este análisis se afirma que la praxis ejecutada por el docente puede afectar de manera positiva o negativa en la ansiedad de los educandos.

En lo que respecta al significado de ajuste, se conceptualiza como la facultad de los seres humanos para manejar correctamente la ansiedad, controlar los impulsos fisiológicos, psicológicos y físicos provocados por las circunstancias que afectan al sujeto.

\subsubsection{Introversión-extraversión}

Este factor está relacionado con la activación del arousal cortical, conectado a los estados de alerta o vigilia, Aguilar y Lluch (1976): afirman que las personas que reaccionan de forma lenta o débil desarrollan conductas introvertidas, contrariamente a los extrovertidos, que responden rápido y demuestran agotamiento.

Estos términos son utilizados en psicología para catalogar a las personas de acuerdo a la dificultad o facilidad para relacionarse con sus semejantes, estas habilidades responden a elementos como la estimulación 
sensorial, percepción y motricidad.

\subsubsection{Calma-excitabilidad}

Condición en que las personas reaccionan o se expresan ante los escenarios que acontecen en el contexto, por ejemplo, Guerra (2015b): asevera que un individuo calmado demuestra tranquilidad ante los hechos y controla la irritación, mientras que una persona excitable es susceptible o agresivo. Por su lado, Catell y Catell (1995c): explican que los sujetos con niveles de excitabilidad elevada suelen ser hiperactivos $u$ obstinados, características perjudiciales para la convivencia armónica en la sociedad.

\subsubsection{Dependencia-independencia}

Interpretando a Catell y Catell (1995d): el factor dependencia expone peculiaridades como la pasividad, se caracteriza porque el sujeto se deja conducir o manejar por el grupo, mientras que en independencia, adoptan el papel de líderes. Del mismo modo, Guerra (2015c): señala que las personas con alto nivel de dependencia sufren al ser expulsados o separados del colectivo, todo lo contrario, a los independientes.

Con base a la teoría expuesta se afirma que la personalidad es un factor determinante para la comprensión de emociones y conductas de los adolescentes en el ámbito educativo, en consecuencia, el docente debe conocer y entender la personalidad de sus estudiantes para orientar, reorientar o adaptar las actividades planificadas en el proceso enseñanza-aprendizaje.

Frente a esto, Herrera, Guevara y Urías (2020): aducen que los profesores desconocen las características de los estudiantes por falta de formación y capacitación, desventaja que enfrenta la educación ecuatoriana en la actualidad. 


\section{Metodología}

La investigación se desarrolló en el año lectivo 2018-2019, acorde con Hernández, Fernández y Baptista (2014): desde un enfoque cuantitativo de tipo no experimental con diseño transeccional correlacional causal, puesto que se recolectó datos en un solo momento para describir relaciones entre dos o más categorías, tal es el caso que con la información se describió las características de la población de acuerdo a las variables y se analizó la correlación entre los factores de personalidad y desempeño académico de los adolescentes.

\subsection{Participantes}

Colaboraron 136 estudiantes adolescentes de tres unidades educativas públicas y dos privadas, ubicadas en dos parroquias rurales en la parte norte de la ciudad de Cuenca, Ecuador.

La selección de las entidades se fundamentó en los siguientes criterios: contexto similar, parroquias rurales, sector norte de la ciudad de Cuenca, noveno año de educación general de básica, estatus socioeconómico medio alto-bajo y bajo, según la clasificación del Instituto Nacional de Estadística y Censos (INEC, 2011): lo cataloga como B, C+ y C-, y establecimientos de sostenimiento fiscal y particular.

\subsection{Instrumentos}

Se manejó el Cuestionario de Personalidad para Adolescentes HSPQ, que mide doce variables de un perfil general y cuatro de factores de segundo orden y perfil. Este instrumento es de fácil aplicación, se les hace a grupos de 30 a 40 estudiantes, en 40 y 50 minutos, para la corrección y obtención de puntaje se tiene una hoja de respuestas con una plantilla. Para la conversión de los resultados existe una tabla de valores numéricos que discriminan y categorizan las variables. 


\section{Resultados (análisis e interpretación de resultados)}

Los datos fueron analizados con el programa estadístico informático SPSS, con un intervalo de confianza de 95\%. Se realizó la prueba T de Student para encontrar la significatividad, análisis de frecuencias para la descripción, correlación de Sperman para evidenciar la relación entre cada uno de los factores de la personalidad y el desempeño académico, finalmente se generó una tabla de contingencia para verificar la relación entre el desempeño académico y el tipo de institución.

Bajo el análisis de los intervalos de confianza se constató que la población estudiada tiene un promedio 13.19 años y el $95 \%$ tiene entre 13.12 y 13.28 , en lo que respecta al promedio presentan una media de 7.67 y oscila entre 7.49 y 7.85 puntos, la clasificación que hace el Instituto Nacional de Evaluación Educativa (INEVAL, 2018a): indica que se encuentran en el nivel medio de la categorización para este estudio y en el fundamental.

\subsection{Análisis descriptivo de la población}

En el análisis de los resultados obtenidos en los factores de la personalidad, se destaca que en reservado-abierto las mujeres tienen puntajes bajos, por lo tanto, exhiben personalidad reservada, callada, alejada, crítica, por el contrario, los hombres presentan personalidad abierta y exteriorizan mejores habilidades sociales.

En emocionalmente afectado-estable, se observa que tanto hombres como mujeres presentan similitud de porcentajes en el nivel bajo, lo que demuestra que pocos tienen menos tolerancia a la frustración y propensión a perder el control, sin embargo, las mujeres lideran el número alto del factor, de lo que se deduce que son más estables y maduras que los hombres.

En calmoso-excitable, los varones resaltan en los tres niveles, lo que representa que son impacientes, exigentes e hiperactivos, frente al porcentaje demostrado por las mujeres, que revela expresividad y actividad reducida. 
En sumiso-dominante, se observa una menor incidencia en el sexo femenino, quiere decir que tienden hacia la sumisión, docilidad y ceder fácilmente, lo contrario del sexo masculino que con mayor porcentaje exponen personalidad dogmática, obstinada y agresiva.

En sobrio-entusiasta, se deduce que las féminas al presentar menor puntaje exhiben características como prudencia, seriedad e inseguridad, mientras que los varones con número más alto demuestran seguridad, confianza y optimismo.

En despreocupado-consciente, los hombres presentan altos porcentajes, se deriva que actúan sin prestar atención a las normas de convivencia y por conveniencia propia, mientras que las mujeres con porcentajes bajos demuestran ser perseverantes, moralistas, sensatas y sujetas a las normas establecidas.

En cohibido-emprendedor, los varones con números altos definen sensibilidad ante las posibles amenazas, mientras que las mujeres con porcentajes bajos expresan espontaneidad y adaptabilidad.

En sensibilidad dura-blanda, se deduce que los hombres, con porcentajes altos, son realistas y rechazan ilusiones, mientras que las mujeres con proporciones bajas demuestran ser impresionables, dependientes y necesitan protección.

En seguro-dubitativo, en los niveles bajo y alto, las mujeres demuestran tendencia a desarrollar actividades grupales y ser activas en este proceso, mientras que los hombres al presentar porcentajes menores tienden a ser reservados e individualistas.

En sereno aprensivo, las mujeres presentan porcentajes más bajos, con lo que muestran inseguridad y preocupación, mientras que los hombres con porcentajes altos demuestran confianza y seguridad en las acciones sociales.

En sociable-autosuficiente, el nivel alto es para las mujeres, se deduce que prefieren tomar sus propias decisiones, mientras que el nivel medio es 
para varones, con lo que exponen mejores habilidades sociales.

En menos más-integrado, el mayor porcentaje es para el sexo masculino, es decir son descuidados con las reglas sociales y siguen sus propias normas, mientras que las féminas con porcentaje bajo manifiestan ser cumplidas con las tareas, tener autodisciplina y controlar su propia imagen.

En relajado-tenso, el porcentaje bajo es para el género femenino, indica que presentan frustración, inquietud o presión en la sociedad, mientras que los varones, al presentar proporciones altas, demuestran tranquilidad y relax ante las circunstancias que viven.

En ansiedad, las mujeres obtienen nivel bajo, en efecto, asumen que la vida es gratificante, entienden la importancia de sus acciones, mientras que los varones al exponer porcentaje alto, presentan incomodidades personales e insatisfacción con los éxitos no alcanzados.

En introversión-extraversión, los porcentajes bajos demostrados por el sexo femenino significa que tienden a ser reservadas e inhibidas a contactos personales, pero pueden realizar trabajos de precisión, mientras que los varones al mostrar porcentajes altos, presentan habilidades sociales eficaces.

En calma-excitabilidad, las mujeres tienen porcentaje bajo, se definen por la sensibilidad blanda, sumisas, sobrias, prudentes, socialmente escrupulosas y poco expresivas, mientras que los varones con porcentaje alto, pronuncian hiperactividad, despreocupación, obstinación, agresividad y problemas para acatar normas.

Finalmente, en al factor general dependencia-independencia, los hombres presentan porcentajes altos, muestran ser pasivos, requieren ser conducidos y tener aprobación del grupo, mientras que las mujeres con porcentajes bajos, expresan iniciativa, atrevimiento y emprendimiento. 


\subsection{Análisis correlacional}

Tabla 1. Análisis correlativo (Rho de Sperman), obtenido de resultados HSPQ.

\begin{tabular}{|l|c|}
\hline \multicolumn{1}{|c|}{ Factores de la personalidad } & Rho de Sperman \\
\hline Reservado-abierto & 0.044 \\
\hline Emocionalmente afectado-estable & -0.20 \\
\hline Calmoso-excitable & -0.21 \\
\hline Sumiso-dominante & 0.80 \\
\hline Sobrio-entusiasta & 0.006 \\
\hline Despreocupado-consciente & 0.2 \\
\hline Cohibido-emprendedor & 0.036 \\
\hline Sensibilidad dura-blanda & -0.008 \\
\hline Seguro-dubitativo & 0.005 \\
\hline Sereno-aprensivo & -0.2 \\
\hline Sociable-autosuficiente & 0.02 \\
\hline Menos-más integrado & -0.032 \\
\hline Relajado-tenso & 0.002 \\
\hline Ajuste-Ansiedad & -0.093 \\
\hline Introversión-Extraversión & -0.074 \\
\hline Calma-Excitabilidad & 0.084 \\
\hline Dependencia-Independencia & 0.08 \\
\hline
\end{tabular}

Fuente: Los Autores (2020).

De acuerdo con los resultados analizados en la tabla 1 , se deduce lo siguiente:

En reservado-abierto, existe correlación de 0.044 , la relación es muy baja y directamente proporcional, los educandos que incrementan el nivel hacia el factor abierto suben el desempeño académico, mientras los que tienen nivel bajo que direcciona hacia la parte reservada bajan en el desempeño académico.

En emocionalmente afectado-estable, la correlación es -0.2, tiene relación baja e inversamente proporcional, los estudiantes con nivel alto hacia el factor emocionalmente estable bajan en el desempeño académico, no obstante, los que tienen nivel bajo dirigido hacia la parte afectada suben el rendimiento escolar.

En calmoso-excitable, el coeficiente de correlación es -0.21 , la relación 
es baja e inversamente proporcional, el alumnado con nivel bajo hacia el factor calmoso sube el desempeño académico, sin embargo, los que exponen niveles altos hacia excitable muestran calificaciones más bajas, cabe recalcar que estas variables se refieren al carácter del estudiante y no tienen relación con calma-excitabilidad que corresponde a la interacción.

En sumiso-dominante el coeficiente de correlación es 0.80 , la relación es muy buena y directamente proporcional, los discentes con nivel alto hacia el factor dominante presentan mejor desempeño académico, como contrapartida, los que muestran sumisión con menores puntajes, tienen calificaciones más bajas.

En sobrio-entusiasta, el coeficiente de correlación es 0.006 , la relación es muy baja o no existe y directamente proporcional, los educandos con nivel alto hacia el factor entusiasta suben en el desempeño académico, antagónicamente, los que tienen puntaje bajo direccionado hacia lo sobrio, presentan calificaciones bajas.

En despreocupado-consciente, el coeficiente de correlación es 0.2 , la relación es baja y directamente proporcional, el estudiantado con nivel alto hacia el factor consciente sube en el desempeño académico, en contraposición, los que tienen menor puntaje dirigido hacia lo despreocupado poseen calificaciones más bajas.

En cohibido-emprendedor, el coeficiente de correlación es 0.036 , la relación es muy baja o no existe y directamente proporcional, los alumnos con nivel alto hacia el factor emprendedor exponen mejor desempeño académico, por el contrario, los que obtienen menor puntaje orientado hacia lo cohibido expresan menores calificaciones, se destaca que este factor no es determinante en el rendimiento conforme a los resultados analizados.

En sensibilidad dura-blanda, el coeficiente de correlación es -0.008, la relación es muy baja o no existe e inversamente proporcional, los estudiantes con nivel alto hacia el factor sensibilidad blanda disminuyen el desempeño 
académico, a diferencia de los que tienen menor puntaje dirigido hacia la sensibilidad dura muestran mejores calificaciones, sin embargo, este factor no es responsable del rendimiento acorde a lo analizados.

En seguro-dubitativo el coeficiente de correlación es 0.005 , la relación es muy baja o no existe y es directamente proporcional, los adolescentes con nivel alto hacia el factor dubitativo suben el desempeño académico, por otra parte, los que tienen nivel bajo encaminado hacia lo seguro presentan promedios más bajos, sin embargo, no es influyente en el rendimiento académico en consonancia a los resultados analizados.

En sereno-aprensivo el coeficiente de correlación es -0.2, la relación es baja e inversamente proporcional, los discentes con nivel bajo orientado hacia la serenidad muestran mejor desempeño académico, no obstante, los que expresan niveles altos encauzados hacia lo aprensivo, bajan el rendimiento académico.

En sociable-autosuficiente, el coeficiente de correlación es 0.02 , la relación es muy baja o no existe y es directamente proporcional, el alumnado con nivel alto orientado hacia autosuficiente presenta mejor desempeño académico, pero los que demuestran puntaje bajo direccionado hacia lo sociable muestran rendimiento escolar más bajo, no obstante, este factor no incide en el rendimiento, expuesto en los resultados examinados.

En menos-más integrado, el coeficiente de correlación es -0.032, la relación es muy baja o no existe e inversamente proporcional, los estudiantes con nivel alto orientado hacia lo más integrado muestra menor desempeño académico, mientras los que presentan menor puntaje conducente hacia lo menos integrado exponen mejor desempeño académico, empero este factor no influye en el rendimiento, como se deduce en los resultados.

En relajado-tenso, el coeficiente de correlación es 0.002 , la relación es muy baja o no existe y al ser positiva es directamente proporcional, los discentes con nivel alto orientado hacia lo más integrado presentan mejor 
desempeño académico, a diferencia de los que tienen menor puntaje encaminado hacia lo relajado obtienen menor desempeño académico.

En ajuste-ansiedad, el coeficiente de correlación es -0.093, la relación es muy baja o no existe e inversamente proporcional, el estudiantado con nivel bajo orientado hacia ajuste expone mejor desempeño académico, por el contrario de los que expresan puntajes altos emplazados hacia la ansiedad, demuestran menor desempeño académico.

En introversión-extraversión, el coeficiente de correlación es -0.074, la relación es muy baja o no existe e inversamente proporcional, los estudiantes con nivel bajo en introversión presentan mejor desempeño académico, por otra parte, los que tienen puntajes altos en extraversión obtienen menores calificaciones.

En calma-excitabilidad, el coeficiente de correlación es 0.084 , tiene relación muy baja o no existe y es directamente proporcional, los alumnos con nivel alto en excitabilidad tienen mejor desempeño académico, al contrario de los tienen menor porcentaje direccionado hacia la calma que muestran desempeño académico bajo.

En dependencia-independencia, el coeficiente de correlación es 0.08 , la relación es muy baja o no existe y es directamente proporcional, el estudiantado con nivel alto en independencia muestra desempeño académico más elevado, a diferencia de los que exponen niveles más bajos orientados hacia la dependencia, revelan desempeño académico bajo.

\subsection{Relación entre tipo de institución y desempeño académico}

Al analizar los resultados de la contingencia, se deduce que los estudiantes matriculados en instituciones privadas presentan mejor desempeño académico $(73,33 \%)$, frente a los de las públicas $(20,88 \%)$, además en las entidades particulares no existe adolescentes con desempeño académico bajo, con relación a lo examinado en las fiscales, que presentan un 
alto porcentaje $(37,36 \%)$, respecto al total de la población estudiada.

\section{Discusión y conclusiones}

La media del promedio general es 7.67 puntos sobre 10, situado en nivel elemental en congruencia con la métrica y clasificación generada por el Instituto Nacional de Evaluación Educativa del Ecuador (INEVAL, 2018b): significa que comprenden e identifican los contenidos impartidos en las asignaturas, sin embargo, lo ideal para una institución educativa es que sus alumnos ingresen al nivel satisfactorio, de tal manera que analicen y relacionen los contenidos enseñados, para lo que deberían alcanzar 8 sobre 10, en consonancia con lo que aportan Bonifaz, Trujillo, Ballesteros, Sánchez y Santillán (2017): el desempeño académico es fundamental para evaluar procesos educativos en las instituciones.

En el perfil de personalidad de las mujeres, predominan los factores caracterizados por la prudencia, tolerancia, responsabilidad, paciencia, docilidad, moral, espontaneidad, dependencia, trabajo grupal, autodisciplina, frustración, satisfacción, inhibición, sobriedad, emprendimiento, coincide con los resultados obtenidos por Benítez, Sánchez y Serrano (2015b): en su investigación sobre indicadores clínicos de la personalidad en la adolescencia en donde afirman que las féminas en esta etapa son preocupadas, inhibidas y prudentes, al igual que Morán, Urchaga, Manga y Fínez (2018b): que exponen que las chicas son resilientes, calladas y se adapten mejor al contexto.

Los hombres en el perfil de personalidad demuestran ser intolerantes, impacientes, agresivos, distraídos, realistas, individualistas, descuidados, relajados, insatisfechos, despreocupados, dependientes, se concuerda con la investigación de Morán, Urchaga, Manga y Fínez (2018c): en donde indican que los varones presentan conductas agresivas y extrovertidas, y con Benítez, Sánchez y Serrano (2015c): quienes aducen que el género masculino se inclina hacia el factor de inseguridad. 
En lo que respecta a la correlación entre los factores de personalidad y desempeño académico se afirma que existe, pero en muy bajo nivel, solamente en el aspecto sumiso-dominante la correlación es fuerte y directamente proporcional con $r=0.80$, en consecuencia, el alumnado con mejores habilidades sociales puede alcanzar promedios sobresalientes en sus calificaciones.

Antagónicamente, esta investigación contradice las aseveraciones presentadas por autores como Poropat (2009): que exponen que el rendimiento académico tiene una fuerte relación con algunos rasgos de personalidad en el Inventario de cinco factores, pues, aunque éstas estudian aparentemente otras variables, después de hacer un análisis de las características se afirma que son semejantes con las planteadas por Catell y Catell (1995e): en el Cuestionario de Personalidad para Adolescentes (HSPQ).

No obstante, los resultados obtenidos se asimilan con la investigación de Cuadra-Peralta, Veloso, Marambio-Guzmán y Tapia (2015): quienes concluyen que existe correlación muy baja o no existe entre los factores de la personalidad y el rendimiento académico, únicamente en responsabilidad obtienen relación baja, $r=0.29$ y directamente proporcional.

El desempeño académico en las unidades educativas privadas es superior al de las públicas, en referencia a esto Benalcázar (2017): dice que se sujeta a que el alumnado perteneciente a instituciones particulares posee un estatus socioeconómico más alto, lo cual garantiza los recursos y medios para mejorar el aprendizaje y no necesariamente porque la educación sea de más calidad; a ello se suma la precariedad en infraestructura, recursos y medios de los establecimientos públicos que también influyen directamente en el proceso enseñanza aprendizaje.

Por otro lado, Osorio y Univio (2017): afirman que la calidad de la educación privada es más eficiente que la pública, puesto que el hecho de tener presupuesto cimentado en el aporte de los padres de familia obliga a la 
entidad educativa particular a cumplir y mejorar aspectos de infraestructura, recursos didácticos, capacitación y cualificación del profesorado, de tal manera que puedan mantenerse con la variedad de la competencia actual, aspectos difíciles de consumar en el sector público.

La personalidad de los adolescentes tiene poca incidencia sobre el desempeño académico, sin embargo, al existir relación alta entre el factor sumiso-dominante y el desempeño académico es importante desarrollar investigaciones referentes a otros constructos que podrían influir en aspectos emocionales, motivacionales o cognitivos.

Desde la perspectiva del deber ético académico vocacional de investigar, forjar actitudes, crear situaciones favorables, atender necesidades, acompañar y orientar a los estudiantes; se recomienda que la planificación de clase elaborada y ejecutada por los docentes contenga estrategias metodológicas de enseñanza-aprendizaje relacionadas con el perfil de personalidad de los adolescentes, aunque está comprobado que no influye determinantemente sobre el desempeño académico, se colige que ésta incide directamente sobre la convivencia armónica generada en el aula.

\section{Referencias}

Aguilar, P. \& Lluch, E. (1976). Estudio experimental del factor de personalidad introversión-extroversión, a través de las manifestaciones vegetativas registradas por medio de la técnica poligráfica. Anuario de Psicología, 1(14), 35-52, e-ISSN: 0066-5126. Recuperado de:

https://revistes.ub.edu/index.php/Anuariopsicologia/article/view/9588/12439

Alarcón, P., Pérez-Luco, R., Wenger, L., Salvo, S., \& Chesta, S. (2018). Personalidad y gravedad delictiva en adolescentes con conducta antisocial persistente. Revista Iberoamericana de Psicología y Salud, 
9(1), 58-74, e-ISSN: 2171-2069. Recuperado de:

https://doi.org/10.23923/j.rips.2018.01.015

Benalcázar, M. (2017). Educación privada versus educación pública en el

Ecuador. Revista Publicando, 4(11.1), 484-498, e-ISSN: 1390-9304.

Recuperado de:

https://revistapublicando.org/revista/index.php/crv/article/view/577/pdf $\underline{398}$

Benítez, M., Sánchez, E., \& Serrano, M. (2015a,b,c). Indicadores clínicos de personalidad en la adolescencia. Psicología desde el Caribe, 32(2), 235-253, e-ISSN: 0123-417X. Recuperado de:

https://www.redalyc. org/articulo.oa?id=21341030004

Bonifaz, I., Trujillo, H., Ballesteros, C., Sánchez, O., \& Santillán, M. (2017). Estilos de vida y su relación con las calificaciones escolares: estudio en Ecuador. Revista Cubana de Investigaciones Biomédicas, 36(4), 1-14, ISSN: 0864-0300; e-ISSN: 1561-3011. Recuperado de:

http://scielo.sld.cu/scielo.php?script=sci arttext\&pid=S0864$\underline{03002017000400006}$

Catell, R., \& Catell, M. (1995a,b,c,d,e). HSPQ. Cuestionario de Personalidad para adolescentes (12-18 años). Sexta edición, ISBN: 84-7174-271-3. Madrid, España: TEA Ediciones, S.A.

Cloninger, S. (2002). Teorías de la personalidad. Tercera edición, ISBN: 97026-0228-9. México: Pearson / Prentice Hall.

Contreras, F., Espinosa, J., Esguerra, G., Haikal, A., Polanía, A., \& Rodríguez, A. (2005). Autoeficacia, ansiedad y rendimiento académico en adolescentes. Diversitas: perspectivas en psicología, 1(2), 183-194, eISSN: 1794-9998. Recuperado de:

https://dialnet.unirioja.es/servlet/articulo?codigo $=2089837$

Cuadra-Peralta, A., Veloso, C., Marambio-Guzmán, K., \& Tapia, C. (2015).

Relación entre rasgos de personalidad y rendimiento académico 
en estudiantes universitarios. Interciencia, 40(10), 690-695, e-ISSN: 0378-1844. Recuperado de:

https://www.redalyc.org/articulo.oa?id=33941643007

Eysenck, H. (1992). A reply to Costa and McCrae. P or A and C-the role of theory. Personality and Individual Differences, 13(8), 867-868, e-ISSN: 0191-8869. Recuperado de:

https://doi.org/10.1016/0191-8869(92)90003-8

Güemes-Hidalgo, M., González-Fierro, M., \& Hidalgo, M. (2017a,b). Pubertad y adolescencia. Adolescere, V(1), 7-22, ISSN: 2695-5474; e-ISSN: 2695-5687. Recuperado de:

https://www.adolescenciasema.org/ficheros/REVISTA\%20ADOLESCE RE/vol5num1-2017/07-22\%20Pubertad\%20y\%20adolescencia.pdf

Guerra, J. (2015a,b,c). Rasgos de personalidad más frecuentes en mujeres guatemaltecas que han sido víctimas de violencia intrafamiliar ejercida por el cónyuge. Tesis de grado. Guatemala: Facultad de Humanidades de la Universidad Rafael Landívar. Recuperado de:

http://recursosbiblio.url.edu.gt/tesiseortiz/2015/05/42/Guerra-Julieta.pdf Hernández, R., Fernández, C., \& Baptista, M. (2014). Metodología de la Investigación. Sexta edición, ISBN: 978-1-4562-2396-0. México: McGraw-Hill / Interamericana Editores, S.A. de C.V.

Herrera, J., Guevara, G., \& Urías, G. (2020). Los estudios de pertinencia desde referentes contextuales: Experiencia desde una maestría en Educación Inclusiva. Revista Scientific, 5(15), 168-190, e-ISSN: 25422987. Recuperado de: https://doi.org/10.29394/Scientific.issn.2542$\underline{2987.2020 .5 .15 .8 .168-190}$

INEC (2011). Encuesta de Estratificación del Nivel Socioeconómico. Ecuador: Instituto Nacional de Estadística y Censos. Recuperado de: https://www.ecuadorencifras.gob.ec/encuesta-de-estratificacion-del- 


\section{nivel-socioeconomico/}

INEVAL (2018a,b). Informe de resultados Nacional: Ser Bachiller. Año lectivo 2017-2018. Quito, Ecuador: Instituto Nacional de Evaluación Educativa. Recuperado de:

https://cloud.evaluacion.gob.ec/dagireportes/nacional/2017-2018.pdf

Jensen, F., \& Nutt, A. (2019). El cerebro adolescente: Guía de una madre neurocientífica para educar adolescentes. ISBN: 978-84-9187-2436. Barcelona, España: RBA Molino.

Malo-Cerrato, S., Martín-Perpiñá, M., \& Viñas-Poch, F. (2018). Uso excesivo de redes sociales: Perfil psicosocial de adolescentes españoles. Comunicar, XXVI(56), 101-110, ISSN: 1134-3478; e-ISSN: 1988-3293. Recuperado de: https://doi.org/10.3916/C56-2018-10

Morán, C., Urchaga, J., Manga, D., \& Fínez, M. (2018a,b,c). La soledad percibida y su asociación con tipos de personalidad en adolescentes españoles. Revista Argentina de Clínica Psicológica, XXVII(1), 44-52, ISSN: 0327-6716; e-ISSN: 1851-7951. Recuperado de: http://dx.doi.org/10.24205/03276716.2018.1046

Osorio, M., \& Univio, J. (2017). La evaluación externa: Tensiones entre la educación pública y la educación privada. Praxis Educativa, 21(3), 58-67, ISSN: 0328-9702; e-ISSN: 2313-934X. Recuperado de: https://doi.org/10.19137/praxiseducativa-2017-210307

Poropat, A. (2009). A meta-analysis of the five-factor model of personality and academic performance. Psychological Bulletin, 135(2), 322-338, ISSN: 0033-2909; e-ISSN: 1939-1455. Recovered from: https://doi.org/10.1037/a0014996

Sierra, J., Ortega, V., \& Zubeidat, I. (2003). Ansiedad, angustia y estrés: tres conceptos a diferenciar. Revista Mal-estar E Subjetividade, 3(1), 1059, e-ISSN: 1518-6148. Recuperado de: https://www.redalyc.org/articulo.oa?id=27130102 


\section{Artículo Original / Original Article}

\section{Luís Leonardo Zambrano Vacacela \\ e-mail: leozamv@hotmail.com}

Nacido en Santo Domingo de los Tsáchilas, Ecuador, el 2

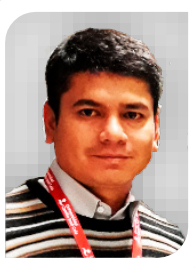
de octubre del año 1981. Doctorando en Ciencias de la Educación por la Universidad Camilo José Cela (UCJC), Madrid, España; Máster Universitario en Psicopedagogía por la Universitat de Barcelona (UB), Barcelona, España; Licenciado en Ciencias de la Educación, mención Ciencias Humanas y Religiosas por la Universidad Técnica Particular de Loja (UTPL), Loja, Ecuador; Docente Autor en la Universidad Nacional de Educación (UNAE), Azogues, Ecuador; Docente Tutor en la Universidad Técnica Particular de Loja (UTPL), Loja, Ecuador; Miembro del Grupo de Investigación Tejidos socio-educativos: Educación, Familias y Comunidad en la Universidad Nacional de Educación (UNAE); Investigador Principal 2 (IP2) del Proyecto de Investigación Neopass@ction, una experiencia colaborativa de videoformación para la profesionalización docente en Ecuador en la Universidad Nacional de Educación (UNAE).

El contenido de este manuscrito se difunde bajo una Licencia de Creative Commons ReconocimientoNoComercial-Compartirlgual 4.0 Internacional 